\title{
An Analysis of the Formation of Regional Linguistic Universals*
}

\author{
Yongming Hong \\ School of Language \\ Xinjiang Normal University \\ Urumqi, China 830054
}

\author{
Jiangmin Zhao \\ School of Language \\ Xinjiang Normal University \\ Urumqi, China 830054
}

\begin{abstract}
Linguistic universality is prevalent in all languages, and its sources are mainly natural ability, pragmatic function, cognitive psychology, diachronic development and language contact. The commonality of language can be divided into three categories: common language, kinship language and regional linguistic commonality. Based on the linguistic generality theory, this paper analyzes the five main ways of regional linguistic common features formation through the analysis of regional linguistic structure and social function variation.
\end{abstract}

Keywords-language contact; regional language; common features

\section{INTRODUCTION}

Language commonality refers to the language category shared by all languages. [1] According to Greenberg's point of view, it can be divided into: absolute commonality and universal tendency, unrestricted commonality and commonality. [2] Comrie divides it into three categories: formal commonality and substantive commonality, containing commonality and non-inclusion commonality, absolute commonality and inclination. [3] There are usually four root causes of language commonality: natural ability, pragmatic function, cognitive psychology and diachronic development. [4] For the latter Bybee believes that diachronic commonality is the law of linguistic evolution, which determines both the possibility of evolution and the directionality. [5] The evolution of language is mainly influenced by the social development and the interaction of various elements within the language. The connection, communication and contact of different societies will inevitably promote the development of language. [6] Therefore, from a diachronic perspective, language contact is also the basis for the commonality of language.

In summary, language commonality can be divided into three categories from the scope of commonality caused by language contact: universal language commonality, relative language commonality and regional language commonality. Regarding the formation of the former two, there is much

Fund Project: This article is a phased achievement of the National Social Science Fund Project---the typological study of Uyghur's sentence (15BYY055) and the National Natural Science Foundation funded project----Chinese-Uyghur Medical and Health Resource Database Construction Research (61562082) discussion in the academic world, and it is self-evident. To this end, the discussion continues on the formation of processes and the process of regional language commonality, that is, how regional languages reach heterogeneous isomorphism under the influence of language contact (different sources, but the structure is the same).

The so-called regional language commonality, also known as the commonality of language alliances, refers to the language categories shared by all languages in a certain region. Shinda Keji believes that the two adjacent languages will gradually become similar even if the language is different; the languages with different language types and similar type characteristics are "language alliances"; sometimes they are collectively referred to as "language circles" regardless of which language they belong to (linguistic area). [7] Whether it is a language alliance or a language circle, its essence refers to regional languages.

The major minority languages in Xinjiang (Uygur, Kazakh) and Xinjiang Chinese dialects (mainly the Lanyin Mandarin) are numerous in terms of pronunciation, vocabulary and grammar, and the language differences between them are also very vague. The relationship between them is the alliance relationship caused by contact, which forms a language alliance and has regional language commonality. Therefore, the Xinjiang Language Alliance can be used as a research object to explore the formation of regional language commonality. According to the status quo of language and historical development, there are five main ways to form the commonality of regional language in Xinjiang:

\section{LANGUAGE CONTACT AFFECTS THE LAW OF THE CIRCLE}

The influence of language contact on the circle law means that in the language alliance, the various languages are ranked from strong to weak and from big to small according to the social function and influence degree; the dominant language becomes the core of the alliance because of the high level of the language field and the degree of influence. Foundation, other languages are on the extranuclear and basic; all languages rely on the form of circle to contact, the closer the circle is, the smaller the influence of language, and vice versa; the core language affects the language of the outer circle, its counter-influence; 
the core language promotes the structural convergence of the outer circle language, and reduces its social function, and finally achieves both language use and language replacement; under the influence of the outer language, the core language will be close to the structure and the social function will be improved. In the language contact between Xinjiang and the Han, the influence of language contact on the circle has always played a role, that is, Chinese is at the center of the contact aggregation, which has an influence on the language of the outer circle; and the language of the outer circle also has an influence on Chinese. Other languages in the aggregator absorb structures from Chinese, and Chinese becomes a basis for the development of these languages. Due to mutual influence, different languages in the polymer body have different structural features from the homologous language of the in-vitro polymerization. Moreover, the commonalities of the various languages of the polymer in the structural features are also expanding, and the boundaries of the system are becoming increasingly weak.

Take the grammatical influence of Chinese on Uyghur as an example: the latter has a more in-depth development of modal particles, and the number of vocabulary words has greatly increased, and the development trend from morphological means to analytical means is obvious. In ancient Uighur, there are only a few restrictions, negations, and doubtful essays, such as: ol ädgü qilün čärüš mu? (Which is good?) Modern Uyghur has not only interrogative modal particles, restricted modal particles, but also imperative tone. Words, votive modal words, exclamatory utterances, indefinite modal particles, indefinite modal particles, etc. Such as: mašina üstinde mügdep qalma jumu (do not sleep when you are in the car).

\section{INTERMEDIATE LANGUAGE SOLIDIFICATION AND GENERALIZATION}

An important influence on language after contact with different languages is the generation of language interference, which is somewhat equivalent to the interlanguage in second language learning. The interlanguage is different from the learner's first language and the target language, but a dynamic language system that gradually moves closer to the correct form of the target language as the learning progresses. Although it is a dynamic system, it is permeable, intractable and repetitive. Therefore, in language contact, this language interference will exist for a long time, accompanied by imperfect bilingual learning, which affects both sides of the contact language within a certain range. Because the interlanguage has both the characteristics of the mother tongue and the characteristics of the target language, it is easy to accept both languages when it spreads. Based on the mechanism of compliance and negotiation, both parties to the communication must use language forms that are acceptable to each other. Therefore, interlanguage naturally becomes the first choice. Through the influence of the interlanguage, the two sides of the contact language can be relatively close in structure and the relationship is ambiguous.

Another aspect of language contact and influence is how the mother tongue affects the target language. The Chinese language used by ethnic minorities in Xinjiang generally bears the imprint of the native language of the nation, that is, the mother tongue in turn affects the target language. This national variant is different from the source of Chinese dialects, but it is roughly the same as the Chinese dialects affected after contact. Despite the structurally affected Chinese dialects, the operators are mainly Han nationalities, but because of the constraints of the language adaptation theory, in order to communicate, their users use the structurally affected Chinese dialects under the impetus of the close motive. The latter is more closely related to the learner's mother tongue, so it is accepted by the learners and becomes a national variant of Chinese. If this national variant is solidified and generalized, it will also lead to a third language that is different from the mother tongue and different from the target language. Further development will lead to similar language appearances in different departments.

\section{DIALECT LINE EXPANSION}

Dialects are local branches of national languages and are the language used by people in local areas. In the contact with Chinese, the national language influences Chinese in two ways: firstly, the Chinese national dialect has a systematic influence on Chinese through the mother tongue, which leads to the formation of dialects. Secondly, the Chinese national dialect is transformed into a Chinese dialect through mother tongue conversion. The two parts of Xinjiang Chinese and North have chosen different basic dialects. Based on this, they have combined other Chinese dialects and other language components, forming the northern and western Mandarins distributed in northern and eastern Xinjiang, distributed in southern Xinjiang and northern. The southern part of the Central Plains Mandarin in Yili, Xinjiang. Judging from the formation history of Xinjiang Chinese dialects, the mutual influence between the Chinese and the Chinese languages plays a decisive role.

After the Qing Dynasty, with the Han immigrants entering the north and south of the Tianshan Mountains, the extensive contact between Chinese and major minority languages, the structure of the Northern Plains of the Lanyin Mandarin and the Southern Xinjiang of the Central Plains were increasingly perfected and the scope of use was also expanding. This ever-expanding dialect line is constantly slamming the major minority languages in Xinjiang, from speech to grammatical structure, semantics to language use. At the same time, we must also see that the Xinjiang dialect itself is the product of the language contact between the people and the people. Therefore, on the basis of the increasingly strict structure and the gradual expansion of the use area, the Chinese dialects of Xinjiang and the major minority language structures are getting closer and closer, and thus forming a language alliance, resulting in similar language features. Uyghur language has absorbed a large number of Chinese dialects in Xinjiang and given certain grammatical changes. Such as: Xinjiang Chinese dialect wo ye (no text form) said that the heart is happy, just right.

\section{Cultural Assimilation}

Cultural assimilation means that due to contact, the cultures of the two peoples are getting closer and closer, and 
eventually assimilation is achieved. The characteristics of the distribution of ethnic groups in China are characterized by "large mixed living and small settlements", which provides a realistic basis for cultural assimilation. There is a parallel and continuous development relationship between cultural assimilation and linguistic assimilation, that is, cultural assimilation process (linguistic behavior process) - guest period (language transfer) - settlement period (language substitution) - transition period (code Conversion) - mixed synchronization (dual language) - assimilation period (residual interference). From the perspective of cultural development characteristics, the process of Xinjiang's folk culture is in a mixed period, and the prominent feature is that bilingual users are more than monolingual users. Dai Qingxia believes that once the language is used (or bilingually used), once it becomes the behavior of the whole nation (or a majority of a nation), the characteristics of the terminology may affect the mother tongue. [20] And this is mainly due to Humboldt's "each language contains a complete system of concepts and imaginations belonging to a certain human group", because it can be familiar with and master the way of thinking in other languages, and then This habit of thinking is injected into the language of the mother tongue, which brings the two languages closer.

The relationship between language and culture is very close. The process of language contact is the process of cultural contact: one is the contact of culture with language load, and the other is the contact of language culture itself. From the perspective of verbal adaptation theory, when communicating with different languages, one of them communicates, and one of them will make their own language closer to the structure of the other language, thus leading to the alienation of their own language structure. When the individual's language behavior is further expanded, it will lead to the bilingual community adopt this communication method. With the development of bilingualism, the structure of this variation will be further expanded, leading to a blurring of the boundaries between different language languages. In the face of non-standard Chinese for bilingual users, Chinese speakers must use Xinjiang dialects or interlanguage similar to their language. When bilingual users use their mother tongue, they will also bring the features of Chinese (especially Xinjiang dialects) into their mother tongue, so that the two languages are similar in structure.

\section{COMMON ANCESTOR REGRESSION}

Ancestral language refers to a common ancestor of several related languages in the same genus-an ancient language, or a communication system that cannot be called "language" at a certain stage of language origin. According to Ramstedt's common Altaic theory, there is a primitive common language in the Altaic minority in the north. This original common language was born in the 6th century BC. It is generally believed that the original common language of the Sino-Tibetan language family-Chinese was formed in the Shang Dynasty more than 3,000 years ago (16th century BC). From the comparison of the basic vocabulary between Middle Chinese and ancient Turkic, the etymological relationship between the two should be homologous. This homology is mainly based on the existence of a large number of related words. For example: the ancient Chinese "filled in" - the ancient Turkic tin, the ancient Chinese "gate" - the ancient Turkic qap and so on.

In the later development of ancient Chinese and ancient Turkic, especially after the 10th century, the Uighurs lived farther away from the Han nationality and inherited the ancient Turkic language, especially after the Islamicization of the latter, the two language structures. The gap between them has gradually increased, moving towards different development paths. At the end of the Qing Dynasty, with the increase of the contact between the people and the Han, the interaction between the Chinese dialects of Xinjiang and the Uighur/Kazakh language was deepening, which showed a return to the common ancestor. The main connotation of the common ancestor regression is that the language is formed under the influence of the inner attraction of the ancestor (primitive language) and the external influence of the society. The formation and evolution of the language family is often in a series of languages. Snowball-style movement is achieved. For the Xinjiang dialects and major minority languages, the final result of "snowballing" is that the structure is getting closer and closer, thus forming an alliance. With the development of society, especially the universalization of language contact, this kind of alliance has a tendency: there are more and more similarities. This situation can be compared with the original language, that is, return to the original language state. It can be seen that the path followed by the Xinjiang Min-han Language Alliance is: homology-differentiation-contact-alliance. Taking the voice as an example, there is a rhyme in ancient Chinese, and in the ancient Turkic language there is also a "homophone" or "corresponding word" that ends the lip of the -p lip. Around the 14th century (or earlier), the sound of the sound disappeared in Chinese. In the simultaneous examination of the Chagatai language and later modern Uyghur language, the words ending in the lip of the $-p$ lip are retained. However, after the Chinese dialects in Xinjiang and the Uyghur language were contacted, the former did not have the influence of the rhyme, and the $-\mathrm{p} / \mathrm{t} / \mathrm{g}$ in the corresponding modern Uyghur syllables disappeared.

Huang Xing believes that if the pedigree relationship of language is not considered to describe the geographical distribution of linguistic structural features, the structural types of Chinese language show a clear tendency of regional convergence, that is, the language distributed in the same region, regardless of whether it has a genetic relationship or not. With some similar structural features, the convergence of such regional structures can be partially explained by the interaction of language contacts. [8] Perhaps this view is the best interpretation of the main purpose of this article.

\section{CONCLUSION}

It is true that although the conclusions about the commonality of regional language in Xinjiang have been more successfully demonstrated, there are not many internal contacts in the formation path. But if we put it in the perspective of contact linguistics, everything can be 
satisfactorily explained. The interlanguage, dialect, cultural convergence, and even the differentiation and development of the original language are inextricably linked with language. Here, the author throws bricks and attracts jade, hoping to get corrections from experts and scholars.

\section{REFERENCES}

[1] John A. Hawkins. Explaining Language Universals[M].Basil Blackwell, 1988: 4.

[2] Greenberg, J.H. Some universals of grammar with particular reference to the order of meaningful elements// In Greenberg[M].1966a: 73-113.

[3] Comrie, B. Review of G. Mallinson and B. J. Blake, Language Typology: Cross-linguistic Studies in Syntax[J]. Language, 1983. (59) 4: 908-911.

[4] Shen Jiaxuan. Review of Hawkins's book "What is Language Commonness" [J]. Foreign Language Teaching and Research, 1991 (4).

[5] John A. Hawkins. Explaining Language Universals[M].Basil Blackwell, 1988: 348

[6] Ye Yisheng, Xu Tongyu. Outline of Linguistics [M]. Beijing: Peking University Press, 1991: 195-199.

[7] Sanada Shinji, Shibuya Hiroshi. Introduction to Social Linguistics [M]. Wang Sumei, translated. Shanghai: Shanghai Translation Publishing House, 2002:74.

[8] Huang Xing. Language Contact and Regional Features of Language[J].National language,2005(3). 